\title{
The method of preparation and use of vasectomized stallions to regulate the sexual function in mares during hippodrome testing
}

\author{
Nursulu Julanova ${ }^{1}$, Orynbay Tagayev ${ }^{2}$, Mardan Julanov ${ }^{1}$, Meruyert Alimbekova ${ }^{1}$, \\ Kanat Koibagarov $^{1}$, Yessengali Ussenbekov ${ }^{1}$, Askar Mautenbaev ${ }^{3}$ \\ ${ }^{1}$ Kazakh National Agrarian University, Faculty of Veterinary Medicine, Almaty, Kazakhstan \\ ${ }^{2}$ A. Baitursynov Kostanai State University, Faculty of Veterinary and Livestock Technology, \\ Kostanai, Kazakhstan \\ ${ }^{3}$ Al-Farabi Kazakh National University, Faculty of Biology, Almaty, Kazakhstan
}

Received July 15, 2014

Accepted November 10, 2015

\begin{abstract}
The objective of this study was to develop a method of preparation and use of vasectomized stallions, and to test them as a factor preventing genital functional disorders in mares during training and hippodrome testing. Thoroughbred mares of English, Arabic and Akhal-Teke breeds owned by horse ranches of the Republic of Kazakhstan were used in the research. Vasectomy techniques were mastered on a slaughtering material, and then field tested on stallions. A series of experiments were set for comparative evaluation of various methods of preparation and use of a vasectomized stallion, resulting in a technique based on removing the front section of sperm ducts in the ventro-caudal portion of the scrotum. The developed method is convenient, safe, and easy to perform in field conditions. The surgery is not time consuming, and there are no postoperative complications. We found a positive effect of the vasectomized stallion on the course of the mares' oestrous cycles, on their performance during hippodrome testing, and their reproductive function. The method reduced the standing heat period, and the mares quickly recovered their performance. Thus, it decreased the time of the mares' elimination from the training schedule and had a positive effect on the performance during hippodrome testing. The use of a vasectomized stallion during training significantly predetermined the course of the mares' reproductive cycle after completion of the hippodrome testing. The results of this study are relevant to the practical needs of sport horse breeding.
\end{abstract}

Horse, scrotum, heat period, prevention, sperm ducts

Reproduction and improving the quality of horses is one of the main objectives of the horse breeding farms. Nonpregnant mares have several sexual cycles within a specific period during the year. Gordon (1983), Livanova (1994), Gavrisha and Kalyuzhniy (1997), Shishkov (1998), Hedberg et al. (2006), and Dzhakupov (2011) indicated that the repetition rate and duration of the cycle are subject to significant fluctuations, depending on the individual characteristics of the mares and environmental conditions.

The signs of heat in mares before competitions make them more difficult to use in sports. According to Shipilov (1977), Le Blanc (1997), Gervek (2009), and Philipova (2012), mares in heat display an increased excitability of the nervous system, signs of sexual reflexes, and they are difficult to manage. All these various behavioural problems are based on the same reason - a strong influence of the sex hormone oestradiol (Kalpokas et al. 2010).

Blue (1987) and Albihn et al. (2003) indicated that the use of a variety of chemical and physical means to suppress oestrus later adversely affects the reproductive function of mares. Therefore, development of effective measures to prevent the functional disorders associated with sexual cycles during training and sport competition is important.

Address for correspondence:

Mardan Julanov

Faculty of Veterinary Medicine

Kazakh National Agrarian University

st. Panfilov 75 m. 10, Almaty, Republic of Kazakhstan 
Some publications suggest that the presence of a male provides normal neurohumoral regulation of the female sexual function (Pavlov 1994; Albihn et al. 2003). However, we were not able to find any advice on the use of a vasectomized stallion in the prevention of genital functional disorders in mares during hippodrome testing.

Given the theoretical and practical importance of this issue, we set the goal to develop a method of preparation and use of vasectomized stallions, and to determine their effectiveness on the mares' course of sexual processes and performance rates during hippodrome testing.

\section{Materials and Methods}

The experiments involved the Akhal-Teke, Arab, and English Thoroughbred roadster breeds of horses of 3-7 years of age, owned by the following enterprises: the "Rahat" and "Akhal-Teke" stud farms, the National School of Advanced Sports Mastery, the Almaty Hippodrome, the agricultural production cooperatives "Plemzavod Almaty" and "Zhorga", the "Bayserke-Agro" limited liability company, the "Beybarys" horse club, the "Akylbay" stud farm, and several private horse farms. These farms were satisfactory in terms of infectious and parasitic diseases. The number of mares involved in the research is listed in Table 1. All animals were physically healthy and had no history of gynaecological diseases at the onset of research.

Table 1. Number of mares of each breed used in the experiment.

\begin{tabular}{ccc}
\hline \multicolumn{3}{c}{ Horse breed } \\
\hline English Thoroughbred & Arabian & Akhal-Teke \\
\hline 124 & 66 & 37 \\
\hline
\end{tabular}

To advance the method of vasectomy, we selected sexually active stallions with no breeding value. The surgery procedure had been practiced on materials from a slaughterhouse (scrotum with the excretory duct of the seminal gland, epididymis and spermatic cord), and then performed on the stallions under local infiltration anaesthesia.

The proposed method of vasectomizing stallions was as follows: the incision was done on the skin and the musculo-elastic membrane on the ventro-caudal surface of the scrotum, $2 \mathrm{~cm}$ away, parallel to its seam (Plate VI, Fig. 1). The tail of the epididymis was pulled to the greatest possible extent by blocking the testis closer to the incision, and then the outer and inner funicular process were dissected (Plate VI, Fig. 2). Simple portion of vas deferens was separated from the fascia (Plate VI, Fig. 3), and $2 \mathrm{~cm}$ of the portion were removed (Plate VI, Fig. 4). The cut of vas deferens was treated with iodine solution, and the upper portion above the cut was ligated to $0.5 \mathrm{~cm}$.

The tail of the epididymis protruding from the wound was washed with a warm and sterile solution of Furacilin at a dilution of $1: 5000$, and then inserted back into the scrotum. The area was dusted with a powder mixture of white streptocide and iodoform at a $1: 1$ ratio. The wound was tightened together with three stitches of the catgut knotted suture. The same procedure was performed with the portion of the second vas deferens. Before using the vasectomized stallions, correctness of the surgery was checked by collecting the ejaculate three times in order to exclude the presence of sperm in the ejaculate.

We conducted three series of experiments for the comparative evaluation of different ways to prepare vasectomized stallions. In the first series (group 1) the surgery was carried out on the base of the scrotum, in the second (group 2) - on the top, in the third (group 3) - on the ventro-caudal portion of the scrotum. Each group consisted of 7 stallions.

The mares were divided into 3 groups with 23 mares in each group in order to determine the methods of using vasectomized stallions. Mares of the first group in oestrus were allowed to mate with a vasectomized stallion daily at an interval of $24 \mathrm{~h}$ until the end of the cycle. Mares of the second group were allowed to mate with a teaser stallion whose penis was retracted back. The stallion's mating was performed similarly to hand breeding. Mares of the third (control) group were not allowed to mate.

The duration of oestrus, the time of drop out from the training schedule, the mares' behaviour during a training session, and their performance achievements were recorded based on the trainer's log book and on our own experimental observation taking into account the opinion of the jockey.

The experimental mares were used on the following equestrian sports: short distance $(1.5-4 \mathrm{~km})$ and long distance (17-30 km) racing, pacing race, show jumping, dressage, and running (30-90 km).

The following equipment was used in the gynaecological examination of mares: the "Eleps" endoscope (Russia), the "MFK" vaginal mirror for mares (Pakistan), and the PU2200-Plus ultrasound apparatus (High Technology Inc., USA).

The research data were statistically processed using the Microsoft Excel program with analysis tool pack for calculating standard deviation. For comparison groups, ANOVA analysis of variance and Tukey's test were used to detect differences at $P<0.05$.

\section{Results}

Access to the dorsal area of the scrotum neck ( $1^{\text {st }}$ series), fixation of the sperm duct, and removal of its initial part were inconvenient. This step of the surgery was also 
time-consuming. Moreover, additional assistance was required to manipulate the lower part of the scrotal septum. At this rate, the procedure of vasectomy lasted $33.4 \mathrm{~min}(\mathrm{~S}=$ $5.41)$. Scrotal oedema was observed in 3 of $7(42.9 \%)$ operated stallions in this group, persisting for some time. Stallions of this group were used in research work 24.6 days ( $=4.58)$ after surgery. The stallions participated in the research for 13.7 months $(\mathrm{S}=7.52)$, and then they were excluded due to reduction of their sexual activity.

Reposition of the testes for extraction through an incision on the caudal epididymitis with sperm ducts was required for access to the initial part of the sperm duct on the top of the scrotum $\left(2^{\text {nd }}\right.$ series $)$. This procedure was time-consuming and also required additional help from assistants. Duration of the surgery in this series of experiments was averaged to $23.0 \mathrm{~min}(\mathrm{~S}=3.21)$, thus consuming $10.4 \mathrm{~min}$ less than the first series, i.e. it was substantially shorter. Two stallions $(28.6 \pm 1.20 \%)$ of this group had a scrotal oedema which resolved within 18-20 days. This group of stallions was set to use in the research 18.3 days $(\mathrm{S}=2.50)$ after surgery. The stallions were used for 37.7 months $(\mathrm{S}=23.42)$.

Caudal epididymis of the testis was easily extracted through the incision and the initial part of the sperm duct was removed in the operative approach on the ventral-caudal portion of the scrotum $\left(3^{\text {rd }}\right.$ series $)$. The surgery was easy and consumed a small amount of time. In addition, the surgeon could perform the operation alone without assistance. Duration of the surgery was $19.4 \min (\mathrm{S}=2.30)$. In this group, one of seven $(14.3 \%)$ stallions had a slight form of scrotal oedema.

The stallions operated by this method were set to use in the research 15.3 days $(\mathrm{S}=1.50)$ after surgery. Vasectomized stallions of the third group were used for a longer time of 87.4 months $(\mathrm{S}=35.81)$.

The extended service of stallions resulted in significant changes in the analysis of variance (ANOVA), to the point of $\mathrm{F}(24.74)=4.29, P<0.001$. Post-effective Tukey's test showed that service duration of the first group of stallions was significantly different from the second and third groups $(P<0.05)$. However, service duration of the second and third groups was not significantly different from each other.

The ANOVA in terms of the stallions' service time after surgery also showed significant differences $(\mathrm{F}=16.024, P<0.001)$. Tukey's test showed that the first group of stallions were used for an extended number of days $(P<0.05)$, whereas the second and third groups were not significantly different from each other in terms of service time.

The ANOVA in terms of monthly service time showed that the stallions that were vasectomized through the ventral-caudal portion of the scrotum were used for a significantly longer period of time $(\mathrm{F}=15.727, P<0.001)$. Tukey's test showed that the third group was significantly different from the first and second groups, whereas the first and second groups did not differ significantly from each other.

As a result of the above comparisons, the method of vasectomy done on ventral-caudal portion of the scrotum proved as the most effective.

Research data proved the effectiveness of using vasectomized teasing stallions to stimulate ovulation and positively regulate the state of mares during the racetrack test, with the mares being calm, light in hand and clearly responding to the commands after the coitus.

On the contrary, mares of the second and third groups were restless in the excitement stage, and hardly controllable with poor athletic performance during the training. They were also irritable and often distracted by a stallion. Mares were growing weary under even a light training load, and some mares displayed lethargy and problems with urination.

The effectiveness of using vasectomized teasing stallions during racetrack training of mares is presented in Figs 5 and 6 . The figures display that daily mounting by a vasectomized teaser during the racetrack training of mares (group 1) significantly reduced the duration of heat by $25.3 \mathrm{~h}(90.1 \mathrm{~h}, \mathrm{~S}=27.02, P<0.05)$. In addition, the mares' drop out time from 


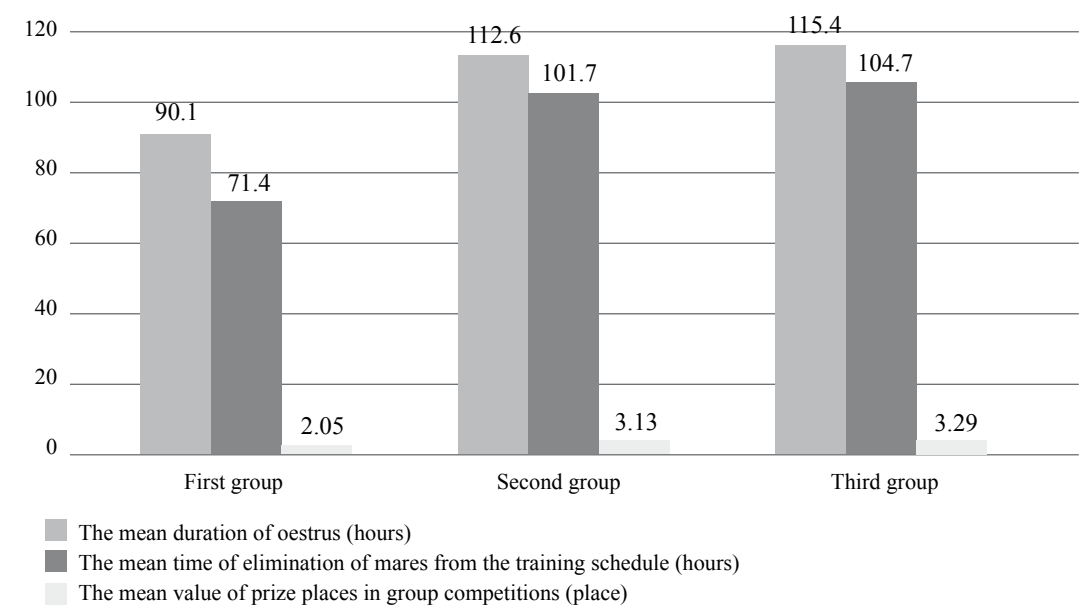

Fig. 5. Analysis of the effectiveness of using vasectomized stallions during hippodrome testing

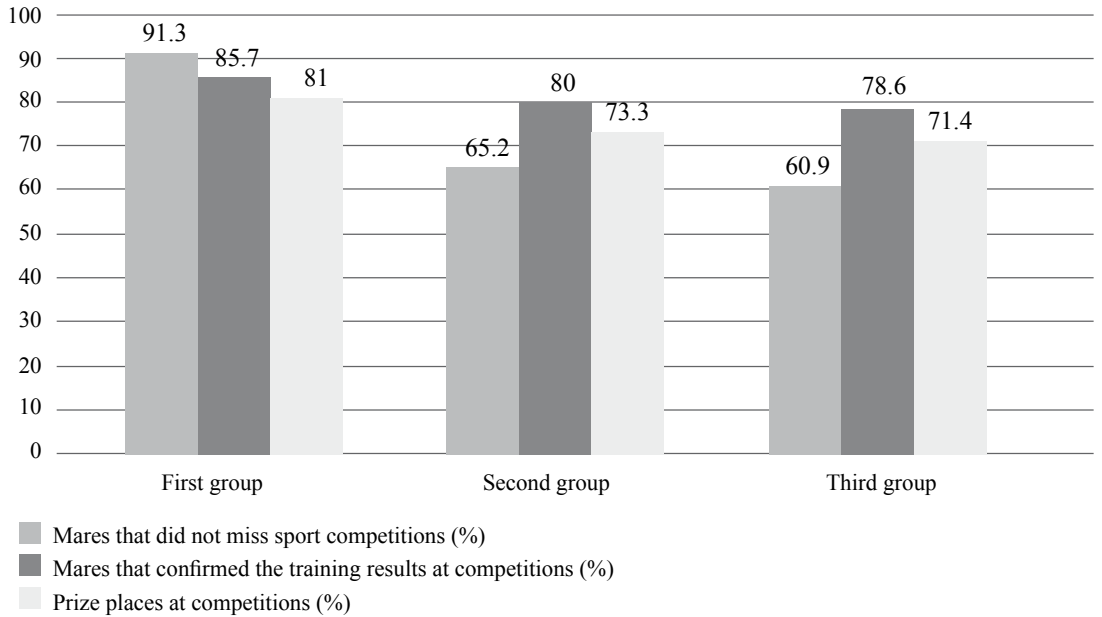

Fig. 6. Analysis of the influence of the vasectomized sample on some indicators during hippodrome testing

the training schedule also significantly decreased by $33.3 \mathrm{~h}(71.4 \mathrm{~h}, \mathrm{~S}=29, P<0.001)$ compared to the control group 3.

Among the mares of group 1, 21 out of 23 animals $(91.3 \pm 6.14 \%)$ did not miss the scheduled sports competitions, which was significantly more than the mares of group 3 , by $30.4 \%(60.9 \pm 10.64 \%, P<0.001)$. At the same time, $85.7 \pm 1.6 \%$ of them held up their athletic performance in competitions, which was by $7.1 \%$ higher than in the control group $(P<0.001)$. Mares of the first group took up more winning places $(9.6 \%)$ compared to the $3^{\text {rd }}$ group $(81.0 \pm 1.79 \%$ compared to $71.4 \pm 1.69 \%, P<0.001)$.

Data on the $2^{\text {nd }}$ group of mares where teaser stallions were used with the penis retracted back did not differ much from those of the control group 3. However, the differences between the variables of mares were significant $(P<0.005)$ when comparing the use of 
stallions capable or incapable of a coitus. For example, when using incapable stallions (group 2), the duration of heat was longer and the number of mares' drop out days from the training schedule was larger $(22.5 \mathrm{~h}, P<0.05$ vs. $30.3 \mathrm{~h}, P<0.001)$ compared to mares using stallions capable of a coitus (group 1). Considerably more mares of the second group $(65.2 \pm 10.38 \%)$ missed sport competitions compared to the first group $(26.1 \%, P<0.05)$ due to heat.

Sport achievements during the training and competitions were the same. However, the performance of second group of mares was significantly less compared to the first group of mares, by $5.7 \%, 80.0 \pm 1.55 \%$ and $85.7 \pm 1.60(P<0.001)$, respectively. The number of winning places in group $2(7.7 \%)$ was much lower than in the mares of group 1 $(73.3 \pm 1.71 \%$ vs. $81.0 \pm 1.79 \%, P<0.005)$. When we used stallions with a retracted penis, the average number of winning places in the group of mares equalled to $3.13 \pm 0.44$, i.e., yielding by 1.08 places to mares which mated with vasectomized stallions during heat $(2.05 \pm 0.30, P<0.05)$.

The use of vasectomized stallions during training significantly influenced the course of the oestrous cycle of mares after the hippodrome training test (Table 2).

Table 2. Analysis of the stallions' influence on manifestation of sexual cycles and genital disorders of mares during the training and hippodrome tests.

\begin{tabular}{lrrr}
\hline Indicators & Group 1 & Group 2 & Group 3 \\
& $\mathrm{n}=23$ & $\mathrm{n}=23$ & $\mathrm{n}=23$ \\
\hline Synchronous sexual cycles, \% & $78.26 \pm 1.98$ & $69.57 \pm 2.21$ & $65.22 \pm 2.28$ \\
& $P<0.005$ & $P<0.001$ \\
Full sexual cycles, \% & $82.61 \pm 1.82$ & $56.52 \pm 2.38$ & $52.17 \pm 2.40$ \\
& $P<0.001$ & & $P<0.001$ \\
Anoestrous sexual cycles, \% & $4.35 \pm 0.98$ & $8.70 \pm 1.35$ & $8.70 \pm 1.35$ \\
& $P<0.01$ & $8.70 \pm 1.35$ & $4.35 \pm 0.98$ \\
Non-responsive sexual cycles, \% & $8.70 \pm 1.35$ & $P<0.01$ & $P<0.01$ \\
& & $17.39 \pm 1.82$ \\
Non-libidinal sexual cycles, \% & $4.35 \pm 0.98$ & $13.04 \pm 1.62$ & $P<0.001$ \\
Anovulatory sexual cycles, \% & $P<0.001$ & $13.04 \pm 1.62$ & $17.39 \pm 1.82$ \\
& & $P<0.05$ & $P<0.001$ \\
Genital pathologies after hippodrome testing, \% & $4.35 \pm 0.98$ & $17.39 \pm 1.82$ & $21.74 \pm 1.98$ \\
& $P<0.001$ & & $P<0.001$ \\
\hline
\end{tabular}

Our results show that using teaser stallions incapable of a coitus was not sufficiently beneficial for stimulating the course of the oestrus cycle in mares after racetrack training tests. The indicators found in groups 2 and 3 confirmed it both by the duration of the oestrous cycle, and by the degree of the cycle's full completion.

Inadequate oestrous cycle in animals of group 1 was manifested in the anoestrous form, and the data were significantly lower compared to the animals of groups 2 and 3 by $4.3 \%$ $(P<0.01)$. Inactive oestrous cycle was observed in all groups of animals, but to a larger extent in mares of groups 1 and 2 compared to group 3 by $4.3 \%(P<0.01)$.

Systematic examination of the mares' genitals revealed no injuries from the use of vasectomized stallions.

The use of vasectomized teaser stallions during the training could affect to some extent the symptoms of genital abnormalities in mares after the racetrack test. For instance, one of the 23 mares in group $1(4.3 \%)$ had a chronic catarrhal vaginitis. In group 2, four mares 
$(13 \%)$ had genital abnormalities, two of them were follicular cysts, one was a chronic suppurative catarrhal endometritis, and one was an ovarian hypo-function. In group 3, five mares $(17.4 \%)$ were recorded with genital abnormalities: follicular cysts in two mares, a hypo-function of ovaries in two mares, and one mare had genital infantilism.

Therefore, animals of groups 2 and 3 had substantially more genital abnormalities than mares of group 1. It should be noted that there were no cases of genital infections or infestations at the mentioned above enterprises, either during the study period or after the research was completed.

\section{Discussion}

As mentioned above, undesirable manifestation of oestrus in mares during the training session and before competition makes it difficult to use them in sports. The frequent occurrence of these "force majeure" circumstances caused a significant economic loss to horse sports clubs and horse ranches. Therefore, development of a method to manage the mare's oestrus cycle during training sessions and the hippodrome test without medication was of interest to breeders.

In our work we used a natural method of managing sexual functions in mares, developing a method of preparation vasectomized stallions. Taking into account the topography of the testes, the cauda of the epididymis and the sperm ducts of stallions, we proposed a vasectomy surgery based on removing the initial portion of the sperm duct at the ventralcaudal portion of the scrotum. This particular area of the scrotum presented a convenient and accessible site for removal of the initial portion of the sperm duct.

This method had fewer complications due to minimum injuries to the blood vessels, sperm ducts, and issues of the scrotum. Also, the cauda of the epididymis stayed intact during the surgery, and a light swelling at the operation site quickly subsided.

Our recommended method allows vasectomized stallions to retain the ability to engage in coitus, which is a fact of exceptional physiological significance. The fact that the vasectomized stallions maintained their sexual activity for a long time can be due to the systematic sexual contact with mares which supported their libido. At the same time, the sperm ejaculated into the cavity of the tunica vaginalis testis was absorbed back, having a stimulating effect on the stallion's body. Thus, the above data show the effectiveness of the developed method.

According to several authors, coitus is a physiological need of the female body during oestrus, as well as a powerful force that affects her behaviour during the cycle. Also, there are publications proving that during coitus the body undergoes processes which trigger the release of the oxytocin hormone responsible for healthy functioning, which calms the animal down (Shipilov VC 1977; Gervek G 2009; Philipova GG 2012). During experiments we observed mares turning to be quiet and easy to manage after coitus, which confirmed the hypothesis. This helped to reduce the number of drop-out days from the training schedule. It also increased the mares' share of participation in competitions, where they maintained the records achieved during the training sessions. The average number of winning places in racing was higher in the group of mares covered by a vasectomized stallion.

In the present study we have shown that preventing mares from having coitus during their oestrous cycle increases their susceptibility to stress during training and hippodrome testing. For example, mares without the possibility to have coitus showed changes in their behaviour manifested by anxiety, aggressiveness and inaccurate performance of the jockey's commands.

Our studies support the hypothesis that the coitus has a beneficial effect on the brain regions responsible for training and memory, and significantly improves responsiveness and concentration. As other authors point out (Shipilov 1977; Pavlov 1994; Hedberg et 
al. 2006; Dzhakupov 2011), the oxygen concentration in the blood rises, thus increasing the activity of the brain. The results of our research show that training mares after having a coitus significantly improved their response and accuracy in performing the commands of the horseman. In addition, all mares were actively used in training, participated in the competition and showed good results.

Our research supports a previous work conducted by Shipilov (1977) in that the neurosexual excitation during the coitus accelerates the completion of oestrus and ovulation. The duration of oestrus in mares which were allowed to mate with a vasectomized stallion was shorter by 30.3 and $33.3 \mathrm{~h}$ than that of mares which were allowed to mate with a stallion incapable of coitus, or of mares which were separated from males, altogether.

It is known that during the coitus a female receives the ejaculate of a male together with testosterone, which reduces the sensitivity of muscles to lactic acid. In our opinion, the fatigue during the training in mares that had no coitus, was associated with the nondelivery of male testosterone.

It is known that zinc increases the resistance of the mucous membrane of the genitals to microbes, bacteria and viruses. Therefore, we believe that the absence of genital abnormalities in mares that had contact with vasectomized stallions were associated with the presence of zinc compounds in the secrets of the accessory genital glands.

Our studies have proved the possibility of using vasectomized stallions in order to normalize the functional state of mares during hippodrome testing. Our proposed method of preparing vasectomized stallions is simple and easy to implement in field conditions. Sexual stimulation of mares by vasectomized stallions had a positive effect not only on the genital function of mares, but also helped them to participate in the training and to achieve high results in competitions. Further research of the hormonal profile of mares will be conducted for the final scientific justification of the effectiveness of the applied method in terms of preparation and use of vasectomized stallions in mares during training sessions and competitions.

\section{Acknowledgements}

We would like to thank the managers, trainers, jockeys and veterinarians of the following enterprises: the "Rahat" and "Akhal-Teke" stud farms, the National School of Advanced Sports Mastery, the Almaty hippodrome, the agricultural production cooperatives "Plemzavod Almaty" and "Zhorga", the "Bayserke-Agro" Limited Liability Company, the "Beybarys" Horse Club, the "Akylbay" stud farm, and several private horse farms of the Almaty and Zhambul regions of the Republic of Kazakhstan for their support and opportunity to carry out the above studies.

\section{References}

Albihn A, Baverud V, Magnusson U 2003: Uterine microbiology and antimicrobial susceptibility in isolated bacteria from mares with fertility problems. J Acta Vet Scand 44: 121-129

Blue MG 1987: Mycoticendometritis in mares. Review and clinical observations. J NZ Vet 35: 181-183

Dzhakupov IT 2011: Veterinarian Obstetrics and Gynaecology (in Russian).. KazATU press, Astana, 167 p.

Gavrisha VG, Kalyuzhny II 1997: Veterinarian Directory (in Russian). Phoenix, Rostov-on-Don, 608 p.

Gervek G 2009: Psychology of the Horse. Temper, Feeling, Behaviour (in Russian). Aquarium, Moscow, 175 p.

Gordon IR 1983: Controlled Breeding in Farm Animals. Pergamon Press, Oxford, 436 p.

Hedberg Y, Dalin AM, Santesson M, Kindahl H 2006: A preliminary study on the induction of dioestrous ovulation in the mare - a possible method for inducing prolonged luteal phase. J Acta Vet Scand 48: 12-18

Kalpokas I, Perdigón F, Rivero R, Talmon M, Sartore I, Vinoles C 2010: Effect of a povidone-iodine intrauterine infusion on progesterone levels and endometrial steroid receptor expression in mares. J Acta Vet Scand 52: 66-68

Le Blanc MM 1997: The equine endometrium and the pathophysiology of endometritis. J Proc Reprod Pathol. Society for Theriogenology 78-84

Livanova TK 1994: Owning a Horse: Advice for Horse Owners (in Russian). Kolos, Moscow, 127 p.

Pavlov VA 1994: Physiology of reproduction in cattle. Rosselhozizdat, Moscow, 208 p.

Philipova GG 2012: Zoopsychology and Comparative Psychology (in Russian). Academia, Moscow, 544 p.

Shipilov VS 1977: Physiological basis for prevention of infertility cows. Kolos, Moscow, 336 p.

Shishkov VP 1998: Veterinaria. The Great Russian Encyclopedia (in Russian), Moscow, 640 p. 
Plate VI

Julanova N. et al.: The Method of ... pp. 63-69

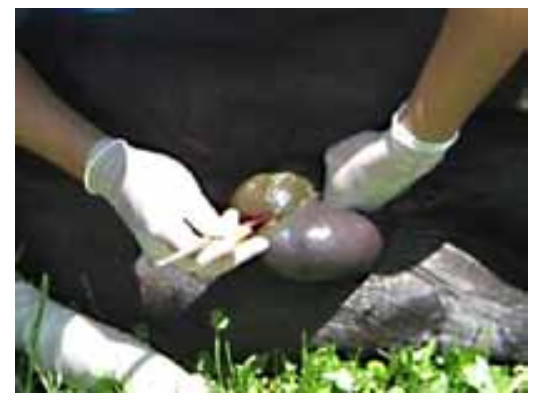

Fig. 1. Scrotal incision

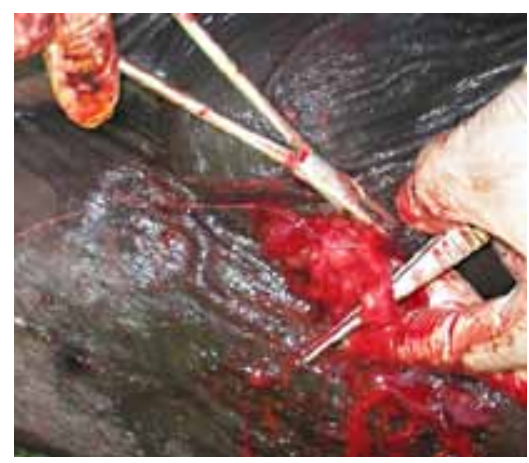

Fig. 3. Deligation of the initial portion of the sperm duct

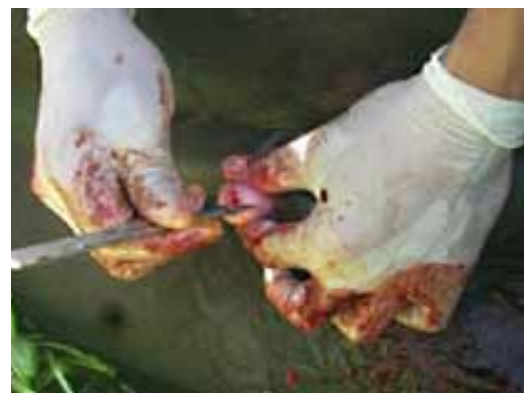

Fig. 2. Dissection of the funicular process

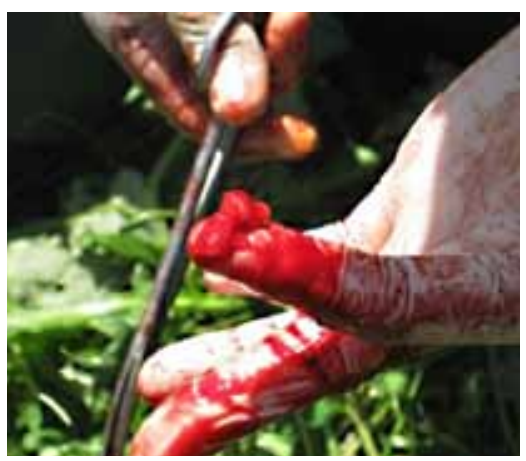

Fig. 4. Removal of the initial part of the sperm duct 